\title{
PABLO NERUDA \\ BIBLIÓFILO Y LECTOR: \\ EL AMOR POR LA VIDA Y EL AMOR POR LOS LIBROS
}

\section{Darío Oses*}

RESUMEN

Este trabajo examina algunos problemas derivados de la relación de Neruda con los libros y la cultura literaria. Expone las posiciones antilibrescas que el poeta enuncia en distintos textos, e intenta un esbozo de conciliación de estas posiciones con el amor por los libros que Neruda no sólo declaró sino también ejerció en sus afanes de coleccionista y en sus lecturas. Asimismo, revisa algunas de sus ideas sobre la poesía y los deberes del poeta.

Palabras daves: Libro, lectura, poesía.

\section{ABSTRACT}

This paper looks at some problems deriving from Neruda's relationship with books and literary culture. It lays open the anti-literary positions that the poet announces in different texts and tries to sketch a conciliation of these positions with the love of books that Neruda not only declared but also exercised in his zeal as collector and in his readings. At the same time some of his ideas about poetry and the obligations of the poet are examined.

Keywords: Books, lecture, poetry.

Recibido: 12.04.2004. Aprobado: 21.06.2004.

^Escritor. Archivo Fundación Neruda. E-mail: dosesbiblio@fundaciónneruda.org 

$\mathrm{N}$

ERUDA NO SOLO fue desde joven un gran lector ${ }^{1}$, sino además un fino bibliófilo, conocedor de obras valiosas y ediciones raras. Sin embargo, más de una vez, en sus escritos, colocó al libro en conflicto con la vida, y se declaró partidario de esta última. En 1954 decía: "Mi generación fue antilibresca y antiliteraria por reacción contra la exquisitez decadente del momento". Y agregaba: "Eramos enemigos jurados del vampirismo, de la nocturnidad, del alcaloide espiritual. Fuimos hijos naturales de la vida"”.

En su "Oda al libro" proclama:

Libro, cuando te cierro abro la vida...

O

Libro, déjame libre.

Y en "Oda a la alegría":

Hoy, alegría, encontrada en la calle, lejos de todo libro acompáñame..."

Y en el poema “Tomás Lago":

Otras gentes se acostaron entre las páginas durmiendo como insectos elzevirianos, entre ellos se han disputado ciertos libros recién impresos como en el foot-ball, dándose goles de sabiduría. Nosotros cantamos entonces a la primavera, junto a los ríos que arrastran piedras de los Andes, y estábamos trenzados con nuestras mujeres sorbiendo más de un panal, devorando hasta el azufre del mundo... ${ }^{3}$

${ }^{1}$ A este respecto, Hernán Loyola apunta: “... Neruda lee mucho en ese tiempo, con ávida y abierta inquietud (no es raro que por las noches devore una o dos novelas, según escribe a Albertina)...” Al decir "en ese tiempo", Loyola se refiere a los años 1925-1926. Ver Loyola, Hernán, "Las dos Residencias", en Neruda, Pablo, Residencia en la tierra, edición de Hernán Loyola, Ed. Cátedra, Madrid, 1987.

${ }^{2}$ Neruda, Pablo, “El rector ha tenido palabras magníficas”. Discurso de Pablo Neruda al donar su biblioteca personal a la Universidad de Chile, el 20 de junio de 1954. En Obras completas de Pablo Neruda. Edición de Hernán Loyola. T. IV. Nerudiana dispersa I, 1915-1964. Ed. Galaxia Gutenberg Círculo de Lectores. Barcelona, 2001.

${ }^{3}$ Neruda, Pablo, “Tomás Lago”, en Canto general de Chile, Canto general, edición de Enrico Mario Santí. Ed. Cátedra, Madrid, 1990. 
Hay otros pasajes, en cambio, en que el poeta exalta al libro, al que considera esencial tanto para la lenta y persistente sedimentación del conocimiento, como para la continuidad de la creación literaria. En su "Oda a la tipografía" alude a esta virtud del libro de desplegar el conocimiento formado a lo largo del tiempo, cuando habla de las letras

\author{
extendiendo \\ el tesoro acumulado, \\ esparciendo de pronto \\ la lentitud de la sabiduría \\ sobre la mesa \\ como una baraja, \\ todo el humus \\ secreto \\ de los siglos...
}

En M emorial delsla Negra escribe:

Los libros tejieron, cavaron, deslizaron su serpentina

y poco a poco, detrás

de las cosas, de los trabajos,

surgió como un olor amargo

con la claridad de la sal

el árbol del conocimiento.

\title{
Y en Confieso que he vivido:
}

Me place el libro, la densa materia del trabajo poético, el bosque de la literatura...

Así, sobre este "humus secreto de los siglos" crecen "el árbol del conocimiento", por una parte, y "el bosque de la literatura", por otra. El libro aparece como el resultado final de una serie de procesos: "la unidad del conocimiento continúa la naturaleza, la inteligencia revela las relaciones más remotas o más simples entre las cosas, y entonces unidad y relación, naturaleza y hombre se traducen en libros"'.

Esta doble visión del libro como letra que seca la vida, por una parte, y por otra como objeto integrador del conocimiento y la creación, aporta algunas claves para determinar las preferencias de lectura de Neruda, las que pueden contrastarse con el examen de sus bibliotecas ${ }^{5}$. En éstas se desplie-

${ }^{4}$ Neruda, Pablo, "El rector ha tenido palabras magníficas".

${ }^{5}$ Se conservan dos bibliotecas formadas por Neruda. La primera es la que el poeta donó en 1953 a la Universidad de Chile. La segunda fue formada con los libros que el poeta tenía, en el momento de su muerte, en sus casas de Santiago, Valparaíso y principalmente en Isla Negra. 
gan las dos virtudes señaladas del libro. El laborioso proceso de formación del conocimiento podría encontrarse en los antiguos libros de historia natural, de exploraciones y viajes, mientras que los clásicos de la literatura universal darían cuenta de la construcción de "la densa materia" artística, literaria, poética. Hay que hacer notar que al poeta no sólo le importa el contenido, sino la conjunción de éste con el libro mismo, como objeto material, exponente de un determinado momento de su propia historia y del desarrollo del arte tipográfico.

Otro problema deriva de la propuesta nerudiana de una poesía antilibresca, antiintelectual, y de una poesía "sin pureza", como él mismo la llamó. A pesar de esto, buena parte de la poesía de Neruda tiene fuentes literarias. Así por ejemplo, Enrico Mario Santí ha rastreado las fuentes bibliográficas de Canto general, y Hernán Loyola, al examinar el puente que salva "el abismo" entre "Serenata" " "Galope muerto", indica algunas lecturas: "Los cuadernos de M alte Laurids Brigge (en versión francesa) de Rilke y la novela M on frérelves, de Pierre Loti; con probabilidad algo de Schopenhauer y de Proust".

El mismo Neruda no sólo hizo poemas a escritores y poetas, sino que entabló diálogos intertextuales principalmente con Whitman, Quevedo, Shakespeare y Melville ${ }^{6}$. Selena Millares ha examinado la herencia de la simbología de Rubén Darío en la poesía amorosa de Neruda ${ }^{7}$. Y el mismo poeta declaró: “... no hay Rubén Darío sin Góngora, ni Apollinaire sin Rimbaud, ni Baudelaire sin Lamartine, ni Pablo Neruda sin todos ellos juntos"8. En un seminario organizado con motivo de sus 60 años, al referirse a su propio aprendizaje del oficio, habla de "la expresión hecha camino, encontrado a través, precisamente, de muchas influencias y de muchos aportes"'.

${ }^{6}$ Hay poemas de Neruda a poetas y escritores como: Federico García Lorca, Rafael Alberti, Miguel Hernández, Juan Larrea, Ramón Gómez la Serna, Juan de Tassis Conde de Villamediana, Alonso de Ercilla y Zúñiga, Jorge Manrique, Francisco Quevedo, Rubén Darío, Antonio Castro Alves, José Martí, Carlos Prestes, Miguel Otero Silva, José González Carbalho, César Vallejo, Oliverio Girondo, Gabriel García Márquez, Ilya Ehremburg, Alejandro Pushkin, Eugenio Evtuchenko, Jules Laforgue, Isidore Ducasse, Jean A. Rimbaud, Louis Aragon, Paul Eluard, Víctor Hugo, William Shakespeare, Nazin Hickmet, Walt Whitman, Howard Fast, y a los chilenos: Joaquín Cifuentes Sepúlveda, Alberto Rojas Giménez, Homero Arce, Angel Cruchaga Santa María, Nicanor Parra, Tomás Lago, Rubén Azócar, Juvencio Valle y Diego Muñoz.

${ }^{7}$ Millares, Selena, "El concepto de lo erótico en Darío y Neruda. Estudio de una simbología común", en Anales de Literatura Hispanoamericana Nº17. Universidad Complutense de Madrid, 1988.

${ }^{8}$ Neruda, Pablo, "Mariano Latorre, Pedro Prado y mi propia sombra", O bras completas.

${ }^{9}$ Emir Rodríguez Monegal apunta: "Como todo gran poeta... Neruda se apoya en la poesía ajena. Si Darío, Sabat Ercasty, Huidobro, la Mistral, Tagore o Whitman marcan sus primeros versos, otros nombres y otros poemas seguirán influyéndolo. Como Shakespeare, como Moliere, como Goethe, como Hugo, Neruda se alimenta de la poesía de su tiempo con la misma naturalidad con que también aprovecha la poesía del fabuloso pasado". En Rodríguez Monegal, Emir, Neruda, el viajero inmóvil. Monteávila Editores, Caracas, 1977.
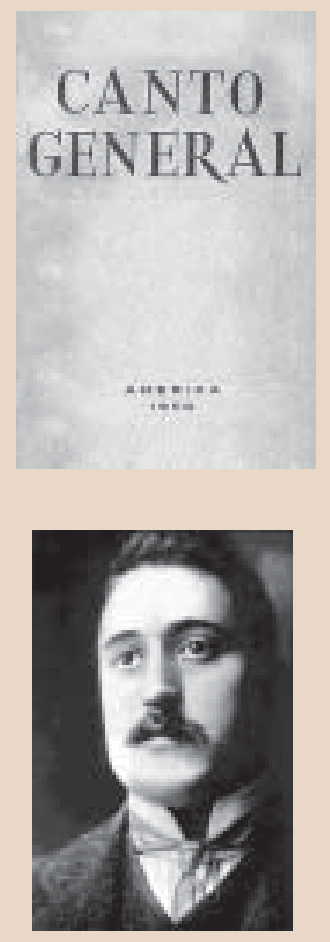

Apollinaire

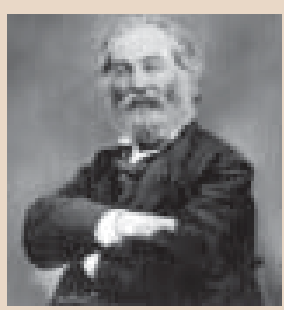

Whitman

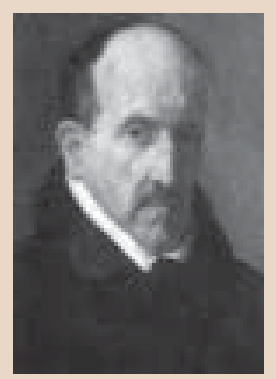

Góngora 


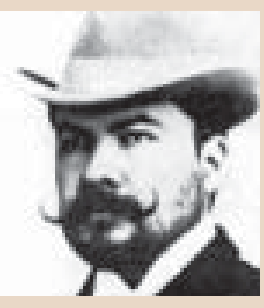

Darío

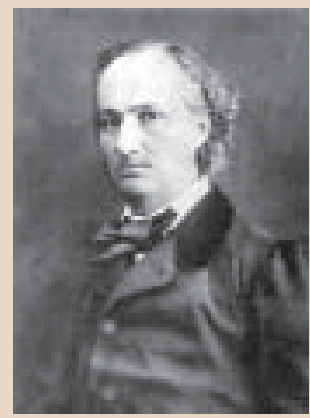

Baudelaire

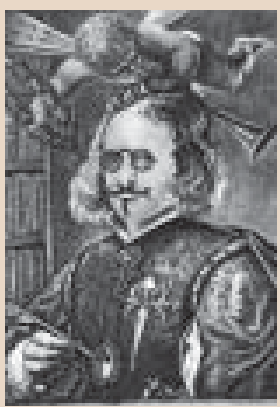

Quevedo
Sin embargo Neruda propuso, con persistente insistencia, una poesía antilibresca, y dedicó varias diatribas contra los poetas atados a los corsés literarios. Asimismo proclamó que no aprendió de los libros cómo hacer poesía, y que tampoco pretendía dejar escritas formas de enseñar a escribirla. Aún así, a lo largo de su obra puede rastrearse un ideario poético. Es interesante observar cómo Neruda va construyendo este ideario a partir de las experiencias de su vida y su trabajo poético. De modo que no sólo declara una posición antilibresca, sino que el mismo hecho de proceder de esta manera se convierte en otra de las manifestaciones de su antiintelectualismo: en lugar de elaborar un manifiesto, o de adherir a alguno ya hecho, Neruda escribe poesía, y de ese trabajo va derivando su reflexión acerca de la poesía y los deberes del poeta.

En octubre de 1935 proponía ya una poesía explícitamente antilibresca. En el prólogo del primer número de Caballo Verde para la Poesía, titulado "Sobre una poesía sin pureza", señala la conveniencia de detenerse a mirar los objetos en descanso: las ruedas, los sacos de carbón, los barriles, los canastos, los mangos de las herramientas. "De ellos se desprende el contacto del hombre y de la tierra como una lección para el torturado poeta lírico".

En los prólogos siguientes desarrolla esta imagen del poeta solitario, solemnemente separado de los otros hombres, y se pregunta, si no habrá llegado ya "la hora de envilecernos", es decir, de hacer una poesía terrenal, que mire "cómo se sostiene el hombre a puro diente, a puras uñas, a puros intereses".

En lugar de la vida, lo "artístico" -como sinónimo, entendemos, de lo libresco"- se apodera de este "poeta lírico".

Contra la "elevación del poeta", Neruda propone la terrenalización o el "envilecimiento" de la poesía; contra el aislamiento del poeta su asimilación a la vida cotidiana, y el trabajo de la poesía hecho con los mismos materiales que se usan en los oficios comunes, y el mimetismo de la poesía con la naturaleza, para tener la misma fuerza que ésta.

Concluye Neruda que "en la casa de la poesía no permanece nada sino lo que fue escrito con sangre para ser escuchado por la sangre", y con esto no sólo propone una forma de escribir poesía sino también de leerla. Su lectura, es decir esta "escucha por la sangre", seleccionaría o aceptaría sólo aquellos textos "escritos con sangre".

En marzo de 1939, en un discurso que leyó en Montevideo, Neruda reiteraba su condición de poeta de la materia: "He querido romper con mi pequeña y desordenada poesía el cerco de misterio que rodea al cristal, a la madera y a la piedra...." Treinta años después, al hablar de su arte poética, sigue proclamando que ésta parte de la materia: "Como poeta carpintero / busco primero la madera / áspera o lisa, predispuesta..." ${ }^{10}$. En 1947, en su

\footnotetext{
${ }^{10}$ Neruda, Pablo, Fin de mundo, en O bras completas, Tomo III, pp. 438-39.
} 
Viaje por las costas del mundo, se autodefine como "poeta natural". Si en los prólogos de Caballo Verde... trazó el perfil del "poeta lírico", en sucesivos textos posteriores va marcando su propia diferencia de "poeta natural" y "material".

Entretanto, sigue completando el perfil de este otro poeta, que él mismo no es. En Canto general, junto con los tiranos, los sátrapas, los oligarcas, los explotadores, los siúticos, los abogados del dólar, y otros cuantos tipos despreciables, aparecen "Los poetas celestes":

Qué hicisteis vosotros, gidistas

intelectualistas, rilkistas, misterizantes, falsos brujos

existenciales, amapolas

surrealistas encendidas

en una tumba, europeizados

cadáveres de la moda,

pálidas lombrices del queso

capitalista... ${ }^{11}$

En este mismo libro hay otras interpelaciones al poeta libresco:

Poeta, buscas en tu libro

los antiguos dolores griegos,

los orbes encadenados

por las antiguas maldiciones,

corren tus párpados torcidos

por los tormentos inventados,

y no ves en tu propia puerta

los océanos que golpean

el oscuro pecho del pueblo..." ${ }^{\prime 2}$

En el poema "El hombre invisible", con el que introduce el primer libro de las odas, Neruda se muestra más benevolente aunque no menos crítico con "el poeta", al que antes ha fustigado. Advierte que adora toda la poesía escrita, pero sigue sonriendo ante el egocentrismo de "los viejos poetas":

él es tan grande

que no cabe en sí mismo,

se enreda y desenreda,

se declara maldito,

lleva con gran dificultad la cruz

de las tinieblas, quías".

${ }^{11}$ Neruda, Pablo, "Los poetas celestes", Canto general, V. "La arena traicionada", II. "Las oligar-

${ }^{12}$ Neruda, Pablo, "Prestes del Brasil (1949)", Canto general, IV, Los libertadores. 
piensa que es diferente a todo el mundo, todos los días come pan pero no ha visto nunca a un panadero (...) y así mi pobre hermano se hace oscuro...

En su discurso “A la paz por la poesía”, de $1953^{13}$, al hablar de su experiencia de escribir Canto general ya decía:

El problema mayor en esos años en la poesía, y naturalmente en mi poesía, ha sido el de la oscuridad y la claridad.

Más adelante:

Nosotros escribimos para gentes sencillas. Escribimos para gentes modestas, que muchas veces no saben leer. Sin embargo, sobre la tierra, antes de la escritura y de la imprenta existió la poesía. Por eso sabemos que la poesía es como el pan, y debe compartirse por todos, los letrados y los campesinos, por toda nuestra vasta, increíble, extraordinaria familia de pueblos.

Hay, entonces, una poesía anterior a la escritura y a la imprenta. Anterior, por lo tanto, al libro y también a la figura del autor. Poesía para todos, porque está hecha por todos. Una de las aspiraciones de Neruda será la de convertirse en la voz de este colectivo humano pretérito y presente: "Yo vengo a hablar por vuestra boca muerta", "Yo estoy aquí para contar la historia".

Más adelante relata:

... fui trabajando en el terreno de la crónica o memorial, que en un principio me pareció pedregoso e inhospitalario. Pero pronto me encontré que esa crónica poética había sido hecha por todos... (el destacado es nuestro).

En sus cuatro libros de odas, Neruda trabajará este canto comunitario, que requiere de un sujeto cantor que renuncie a vivir consigo mismo y para sí mismo, como el egocéntrico poeta lírico tradicional; un poeta que se haga invisible, que deje que su ego se esfume, para cantar, vivir y ser hombre con los otros hombres, buscando, además, una legibilidad popular, una claridad amplia.

En “A la paz por la poesía” Neruda agregaba:

Nuestras plantas, nuestras flores deben por primera vez ser contadas y cantadas. Nuestros volcanes y nuestros ríos se quedaron en los secos espacios de los textos. Que su fuego y su fertilidad sean entregados al mundo por nuestros poetas. Somos los cronistas de un nacimiento retardado...

${ }^{13}$ Neruda, Pablo, “A la paz por la poesía”. Discurso leído en el Teatro Caupolicán, el 26 de junio de 1953, ante la Asamblea Plena del Congreso Continental de la Cultura, en O bras completas. 
La poesía auténtica aparece, así, como un bien y una creación colectivos, y tiene la misma vida que el paisaje natural. Si este paisaje fue secado por los textos, los poetas deben devolverles su "fuego y fertilidad". La poesía debe diferenciarse, por lo tanto, de los textos y los libros que resecan y apagan.

Esta poesía, "escrita con la sangre", debe entonces vivificar la historia y la naturaleza.

En Confieso que he vivido, el poeta escribe:

Me place el libro (...) pero no las etiquetas de las escuelas. Quiero libros sin escuelas y sin clasificar, como la vida.

Para Neruda habría, entonces, en términos muy generales, al menos dos tipos de textos y dos tipos de lecturas: aquéllas directas, sencillas, transparentes, legibles, naturales y cercanas a la vida -éstas se encontrarían en el área de la luz-, y las otras, las oscuras, las que requieren de la mediación de aparatos analíticos; lecturas dirigidas por escuelas, libros encasillados por las clasificaciones.

La poesía, capaz de reproducir "el fuego y la fertilidad" de la naturaleza, merece una lectura afín con esa potencia reproductora: "en la casa de la poesía no permanece nada sino lo que fue escrito con sangre para ser escuchado por la sangre".

\author{
Libros sagrados y sobados, libros \\ devorados, devoradores, \\ secretos, \\ en las faltriqueras: \\ Nietzsche, con olor a membrillos, \\ y subrepticio y subterráneo, \\ Gorki caminaba conmigo.
}

En estos primeros versos del poema "Los libros", de M emorial de Isla Negra, aparece esta serie de dobles condiciones del libro: sagrado y profano; devorado y devorador; secreto, pero en las faltriqueras, donde se llevan las cosas de uso corriente; el oscuro Nietzsche devuelto a la corriente cotidiana por su proximidad con el membrillo, y finalmente Gorki, por un lado "subrepticio y subterráneo", pero caminando al lado del poeta.

En el discurso del Premio Nobel, en diciembre de 1971, reaparecen ideas expuestas en "A la paz por la poesía", de 1953. El poeta vuelve a usar las metáforas del pan y de la luz en relación con la poesía.

Dice:

Yo no aprendí en los libros ninguna receta para la composición de un poe- 
ma; y no dejaré impreso a mi vez ni siquiera un consejo, modo o estilo para que los nuevos poetas reciban de mí alguna gota de supuesta sabiduría ${ }^{14}$.

Neruda había relatado antes, en ese mismo discurso, el viaje a caballo que hace a través de la Cordillera de los Andes, para salir clandestinamente del país, a principios de 1949. Allí conoció la elemental fraternidad entre los hombres, y experiencias como ésa -más que tratados o elaboraciones librescas- fueron las que le enseñaron lo que es la poesía.

"En aquella larga jornada encontré las dosis necesarias a la formación del poema" -dice Neruda-. La poesía aparece aquí como un gran ejercicio integrador de la realidad y los sueños, del hombre y la naturaleza, de las intimidades individuales con las colectivas. El flujo entre las dimensiones personales y colectivas termina por confundirse en esta epifanía. Neruda dice:

... no sé, después de tantos años, si aquellas lecciones que recibí al cruzar un río vertiginoso, al bailar alrededor del cráneo de una vaca, al bañar mi piel en el agua purificadora de las más altas regiones, digo que no sé si aquello salía de mí mismo para comunicarse después con muchos otros seres, o era el mensaje que los demás hombres me enviaban, como exigencia o emplazamiento.

De todo esto Neruda concluye: "el poeta debe aprender de los demás hombres", y luego retoma la metáfora del panadero: "el mejor poeta es el hombre que nos entrega el pan de cada día: el panadero más próximo...”

En el primer discurso de Estocolmo, el que pronuncia Neruda a nombre de todos los laureados de ese año, vuelve al tema del continente oscuro que busca la luz, y al de la pertenencia del poeta a la "multitud humana" que lo hace sentirse rodeado de una "presencia invisible" y la necesidad de que la poesía se incorpore "al trabajo común de cada día y de todos los hombres"15.

La poesía -dotada de un valor instrumental para la comunicación entre los hombres- pasa a formar parte de este caudal de la vida diaria, y el poeta es, por una parte un trabajador, como tantos otros, y es también parte de una continuidad que va más allá de él. En el discurso en que agradece el Nobel a título personal, dice:

\footnotetext{
${ }^{14} \mathrm{Tal}$ vez lo más cercano a un consejo sea su propia experiencia en la composición de un poema, que se encuentra en "Artes poéticas" I y II, en Fin de mundo. Aquí Neruda, entre otras cosas, retoma la metáfora del poeta panadero.

${ }^{15}$ Neruda, Pablo, "La presencia invisible". Discurso para agradecer el Premio Nobel en nombre de todos los laureados del año 1971, en Neruda, Pablo, La poesía no habrá cantado en vano. D iscursos de Neruda con ocasión del Premio Nobel de Literatura. Libros del Ciudadano. Lom Ediciones, Santiago, 2001.
} 
Cada uno de mis versos quiso instalarse como un objeto palpable; cada uno de mis poemas pretendió ser un instrumento útil de trabajo: cada uno de mis cantos aspiró a servir en el espacio como signos de reunión, donde se cruzaron los caminos, o como fragmentos de piedra o de madera en que alguien, otros, los que vendrán, pudieran depositar los nuevos signos (destacado nuestro).

El poeta reunió una doble herencia literaria universal: la de sus libros como objetos materiales, y la de sus lecturas, y la traspasa "a los nuevos poetas de América", como dice en su poema "Testamento", de Canto general, donde los libros que él deja son el telar para hilar "las significaciones del mañana".

De modo que en esta "expresión hecha camino" de que hablaba Neruda en 1964 no sólo están los aportes que recibe, sino también los "nuevos signos". El camino continúa más allá del mismo Neruda quien, al regresar a Chile en 1972, dice: "Porque la vida, la lucha, la poesía, continuarán viviendo cuando yo sea sólo un pequeño recuerdo".

Confiamos en haber esbozado una propuesta acerca de cómo podrían resolverse las tensiones entre las posiciones antilibrescas que Neruda enuncia, declara y ejerce tanto en su propio trabajo poético, como en su vida, con la también declarada admiración por los libros y por diversos autores de la literatura universal. Una posibilidad de conciliación de estos dos amores: a los libros y a la vida, que el mismo poeta percibe en algunos momentos como incompatibles.

Por último, es interesante observar que al revisar las ideas sobre la poesía y los deberes del poeta que Neruda expone en diversos escritos, entre 1935 y 1972 , se comprueba cierta continuidad que contrasta con los cambios y rupturas que la poesía misma de Neruda experimenta en el mismo período.

\section{BiBLIOGRAFÍA}

Loyola, Hernán. 1987. “Las dos Residencias”, en Neruda, Pablo, Residencia en la Tierra, edición de Hernán Loyola. Madrid: Ed. Cátedra.

Millares, Selena. 1988. "El concepto de lo erótico en Darío y Neruda. Estudio de una simbología común", en Anales de literatura hispanoamericana $\mathrm{N}^{\circ} 17$. Universidad Complutense de Madrid.

Neruda, Pablo. 1990. Canto general. Edición de Enrico Mario Santí. Madrid: Ed. Cátedra, Letras Hispánicas.

. 2001. "El rector ha tenido palabras magníficas". Discurso de Pablo Neruda al donar su biblioteca personal a la Universidad de Chile, el 20 de junio de 1954. En Obras completas de Pablo Neruda. Edición de Hernán Loyola. T. IV. Nerudiana dispersa I, 1915-1964. Barcelona: Ed. Galaxia Gutenberg Círculo de Lectores. 
2001. "La Presencia invisible”. Discurso para agradecer el Premio Nobel en nombre de todos los laureados del año 1971, en Neruda, Pablo, La poesía no habrá cantado en vano. Discursos de Neruda con ocasión del Premio Nobel de Literatura. Santiago: Libros del Ciudadano. Lom Ediciones,

2001. "A la paz por la poesía”. Discurso leído en el Teatro Caupolicán, el 26 de junio de 1953, ante la Asamblea Plena del Congreso Continental de la Cultura, en O bras completas. Barcelona: Ed. Galaxia Gutenberg Círculo de Lectores. $887 \mathrm{pp}$.

- 2001. “Mariano Latorre, Pedro Prado y mi propia sombra”, en O bras completas. Barcelona: Ed. Galaxia Gutemberg Círculo de Lectores.

Rodríguez Monegal, Emir. 1977. Neruda, el viajero inmóvil. Caracas: Monteávila Editores.

Sicard, Alain. 1981. El pensamiento poético de Pablo Neruda. Versión en español de Pilar Ruiz Va. Madrid: Ed. Gredos, Biblioteca Románica Hispánica.

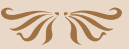

